\title{
A Study on Simulation of Single Sailing Training System
}

\author{
Xiaofeng Chang ${ }^{1}$, Xin Luan², Guojia $\mathrm{Hou}^{2,3^{*}}$ \\ ${ }^{1}$ Teaching Center of Fundamental Courses, Ocean University of China, Qingdao, 266100, China \\ ${ }^{2}$ College of Information Science and Engineering, Ocean University of China, Qingdao, 266100, China \\ ${ }^{3}$ College of Information Engineering, Qingdao University, Qingdao, 266071, China
}

\begin{abstract}
The single sailing simulation system is introduced in this paper based on a large amount of data from the experiment. Combining traditional method with the fuzzy control, a new method of routes planning is put forward. Relying on simulating the system using MATLAB tool, we can find the optimist degree and routes of the sailing using the shortest time reach the finish line in theory. The planning routes can be used to direct the sailing through coordinate conversion. Modelling the single sailing and marine environment based on Virtual Reality Technology, simulate the sailing. The Simulation results prove that the proposed method in this paper is helpful for the sailing training
\end{abstract}

Keywords-single sailing; optimization path; simulation; virtual reality.

\section{INTRODUCTION}

Modern sailing originates from Netherlands. The sailing is a sport combined by entertainment, ornamental, exploration and athletics. It is motivated by natural wind and sails on the ocean. China is a nation that has long history of sailing cultural. Even if the single sailing has short history, our country has made a great progress along with more attention paid on it. It is really significant to do more research on single sailing training, such as path optimization and weather forecasting.

The sailing needs wide space to compete on the ocean, which is $1-2 \mathrm{~km}$ away from the coast. The Olympic sailing competition usually adopts trapezoid traces, as shown in Figure.1. The competition field is an equilateral triangle constructed with three buoys. Each edge is no less than 22.5 sea miles. The starting and finishing line are both virtual line which is 100-200 meters wide, and the width decreases as the amount of the sailings cut down. Another way to calculate is using the amount of the boat multiple the length of the boat firstly, and then using the number multiple 1.25 meters. The starting and finishing lines are parallels. The first buoy and the wind direction form 90-degree angle.

Because of the environment condition and the competition field is always varied, the field is settled based on the real-time environment. The preparation work should be finished in 5 minutes before the competition started. The sailing trace should be combined by against wind, horizontal wind, tailed wind. It will test the athletics overall abilities, not just fortune.

Sailing is restricted by the field, the equipment and so on. It is a complicated game which is affected by many aspects. To improve the quality of the training system, we use MATLAB tool to simulate all the influence factors in the sailing process. We use the traditional algorithm combined with artificial programming methods to calculate the optimized sailing trace. Based on the simulation and ocean experiment, the result can guide the athletics in an effective way.

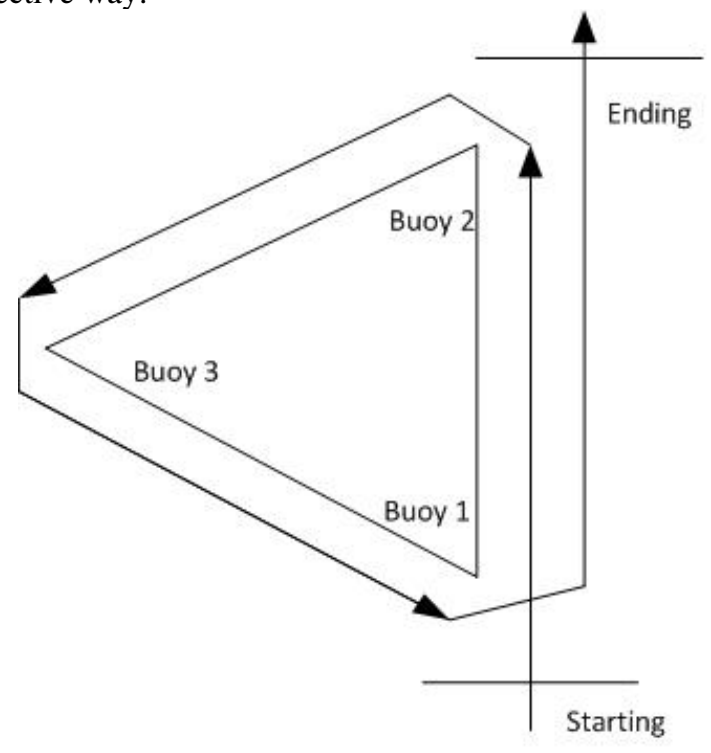

Figure 1. Sailing route of sailboat

\section{FORCE ANALYSIS AND BEST SAILING ANGLE}

The sailing is composed by five sections, hull, masts, sails, the rudder and the central board. As shown in Figure. $2 \mathrm{a}$, the hull is the main portion. The hull's size is different in the kinds of the sailings. The mast is used to stick sails. The sails received wind from the ocean which is the power source of the sailing. The rudder is like the steering wheel in car. The central board is used to balance sailings. 


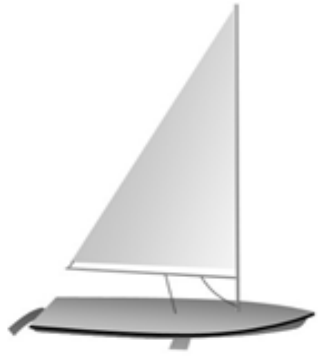

(a)

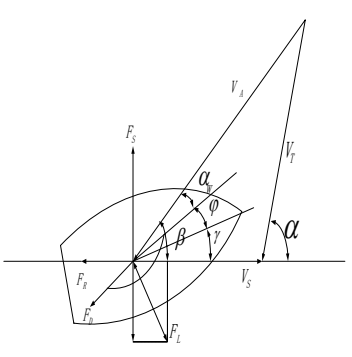

(b)
Figure 2. (a) The sailboat structure, (b) Principle diagram of force

The power of the sailing is collected from wind. When the wind blows across the sails, two sides of the sail receive different force based on Bernoulli Effect. We can classify the wind into three kinds, the real wind, the sailing wind and the relative wind. The real wind is the wind speed tested on the mainland. The sailing wind is the opposite direction wind which is equal to sailing speed. The relative speed is the vectorial resultant of the real wind and the sailing wind. We assume that the sailing sails along right direction as shown in Fig. 2b. The real wind $V_{T}$ and the direction form an angle $\alpha$. The speed of the sailing is $V_{S}$. The direction and the sails form an angle $\gamma$. The relative wind speed $V_{A}$ and the sailing direction form an angle $\beta$. When wind blow across the sailing, based on Bernoulli Effect, the pressure on the slow speed side is larger than the other side. The pressure forms a force $F_{L}$, which is vertical to the sails. The stream forms a force $F_{D}$, the aerodynamic lift and aerodynamic drag form the motivation power [1]:

$$
F=F_{L} \sin \beta-F_{D} \cos \beta
$$

The aerodynamic lift and aerodynamic drag form the horizontal force, the force is:

$$
F_{t}=F_{L} \cos \beta+F_{D} \sin \beta
$$

The aerodynamic lift coefficient $C Y$ and aerodynamic drag coefficient $C X$ are lift and drag force. The sails lift force and the drag forces are as below:

$$
Y=\frac{1}{2} \cdot \rho \cdot c_{y} \cdot A \cdot V_{A}^{2}
$$

In the formula, $Y$ is the lift force, $X$ is the sails' drag force, the $\rho$ is air density, $A$ is the area of the sails, $V$ is the relatively wind speed.

The relatively wind blow the sails in speed $V_{A}$ with angle $\alpha$. The relatively wind and the direction force an angle $\beta$. The sails get the force

$$
F_{t}=\rho_{\text {air }} \cdot A \cdot V_{a}^{2} \cdot \sin \alpha
$$

$F_{t}$ can be separate into forward force $F_{r}$ and vertical force $F_{h}$ [2]. When the sails get the balance force, the sailing will get uniform motion. The acceleration of the sailing is really fast which can be ignored. So the wind force is equal to the drag force, as below:

$$
V_{b}^{2}=F_{t} \cdot \sin (\beta-\alpha)=\rho_{\text {air }} \cdot A \cdot V_{a}^{2} \cdot \sin \alpha \cdot \sin (\beta-\alpha)
$$

The speed of the sailing can be defined as [3].

$$
V_{b}=\sqrt{\frac{\rho_{\text {air }} \cdot A \cdot \sin \alpha \cdot \sin (\beta-\alpha)}{C}}
$$

Where $C$ is the drag force coefficient. The ship speed in $L$ direction is

$$
V_{B M}=V_{b} \cdot \cos \omega
$$

The sailing time is

$$
t=\frac{L}{V_{B M}}=\frac{L \cdot \sqrt{C}}{\sqrt{\rho_{\text {air }} \cdot A \cdot V_{A}^{2}}} \cdot \frac{1}{\sqrt{\sin \alpha \cdot \sin (\beta-\alpha) \cdot \cos \omega}}
$$

In order to minimize the time, we could use the fmincon function to do that [4]. We separate the sailing period into each parts and optimize it [5].

TABLE I. RELATIONSHIP BETWEEN THE ANGLE OF WIND, HEADING AND ROLLING

\begin{tabular}{ccccc}
\hline $\begin{array}{c}\text { Wind } \\
\text { angle } \\
\theta\end{array}$ & $\begin{array}{c}\text { Rolling } \\
\text { angle } \\
\alpha\end{array}$ & $\begin{array}{c}\text { Angel } \\
\text { between } \\
\text { sail and } \\
\text { (degree) }\end{array}$ & $\begin{array}{c}\text { Heading } \\
\text { angel } \\
\omega\end{array}$ & $\begin{array}{c}\text { Minf } \\
(\alpha, \\
\text { heading }\end{array}$ \\
$\begin{array}{ccc}\boldsymbol{\alpha} \text { (degree) } \\
\text { (degree) }\end{array}$ & $\beta$ ) \\
\hline 0 & 24 & 24 & 48.0 & 3.6742 \\
15 & 28.7 & 28.7 & 42.4 & 2.8199 \\
30 & 33.5 & 33.5 & 37.0 & 2.2686 \\
45 & 38.6 & 38.6 & 32.2 & 1.8942 \\
60 & 43.8 & 43.8 & 27.6 & 1.6303 \\
75 & 49.2 & 49.2 & 23.4 & 1.4394 \\
90 & 54.7 & 54.7 & 19.4 & 1.2990 \\
105 & 60.4 & 60.4 & 15.8 & 1.1953 \\
120 & 66.2 & 66.2 & 12.4 & 1.1190 \\
135 & 72.1 & 72.1 & 9.2 & 1.0646 \\
150 & 78.0 & 78.0 & 6 & 1.0280 \\
165 & 84.0 & 84.0 & 3 & 1.0069 \\
180 & 90 & 90 & 0 & 1 \\
\hline
\end{tabular}

\section{SAILING PATH PLANNING BASED ON FUZZY CONTROL}

The traditional control is an accurate control. This method needs to analyze the data and description accurately and it uses unified formula to control [6]. The force analysis of sailing is very complex and always changeful. We should use GPS to control the sailing paths [7].

The path-planning question can be divided into two kinds based on the environment. The first is the pathplanning based on model. All the information can be accessed in this kind. The other is the path-planning based on the sensors. The information of the environment can be access partly or nearly unknown [8].

Based on the fuzzy control planning, we can utilize the formula shown above as the knowledge storage. We can 
make out that, when the wind angle larger than $90^{\circ}$, the function changes a little. So, in this condition, the sailing sails straight forward without caring other information. The function changes a lot when the wind angle is less than $90^{\circ}$. Then, the optimized sailing angle has to be considered [9].

Based on force analysis, when the relatively wind angle not equal to $180^{\circ}$, the sailings could always move straight forward. So, when the wind angle is more than $90^{\circ}$. The sailing moves following wind. In this condition we consider optimizing the forward speed. When relatively wind angle is less than $90^{\circ}$, the sailing moves against the wind. In this condition, the sailing moves as shown in Figure.3.

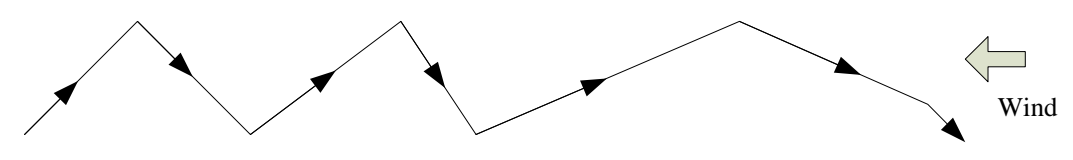

Figure 3. Sketch map of the sailing trace which is like the Chinese character of 'Zhi' when sailing against wind.

Based on the optimized angle, we can design our fuzzy control algorithm. We use the member function [10].

$$
Z(x, y)=\mid \begin{gathered}
0, \sqrt{\left(x-x_{t}\right)^{2}+\left(y-y_{t}\right)^{2}} \geq R \times g(\vec{V}, \vec{D}) \\
1-\frac{\sqrt{\left(x-x_{t}\right)^{2}+\left(y-y_{t}\right)^{2}}}{R \times g(\vec{V}, \vec{D})}, \text { else }
\end{gathered}
$$

Where $\left(x_{t}, y_{t}\right)$ is the target, $R$ is larger than 0 . We select $R=L, L$ is sailing distance, $\vec{V}$ is the speed, $\vec{D}$ is the vertical distance $(x, y)$ away from the target $\left(x_{t}, y_{t}\right), g$ is direction coefficient, we can select $g>0$ based on different situations, g is $\vec{V}$ and $\vec{D}$ angle's reduce function.

$$
g(\vec{V}, \vec{D})=1+\lambda \cos \varepsilon
$$

Here $0<\varepsilon<90^{\circ}$, It is the angle formed by $\vec{V}$ and $\vec{D}$. We select $E V, T$ is the sailing's finishing time across the $\mathrm{k}$ area [11].

$$
\begin{gathered}
E V=\tau_{1} T+\tau_{2} Z(x, y) \\
T=L /(n \cdot V)
\end{gathered}
$$

$\mathrm{V}$ is the separate speed on the shortest path.

$$
V=V_{y}=V_{B} \cos \psi
$$

$Z(x, y)$ is the member function, $\tau_{1}, \tau_{2}$ are weighting coefficients. During competition, we are looking forward the shortest time, so $\tau_{1}>0, \tau_{2}<0$ Through knowledge we can set the parameters as $\tau_{1}=0.9, \tau_{2}=-1.08$, $\lambda=0.7[12]$

\section{SimULATION RESUlTS}

The sailing competition changes a lot, including so many parameters, so the artificial path planning can fit all the conditions. The fuzzy path planning algorithm is a kind of artificial path planning algorithm. It doesn't need the accurate math function. We can use the fuzzy control method instead of it. It can fit the changing environment. Fuzzy control uses control method to describe the relationship between the parameters. That is to say that the fuzzy control function doesn't need the accurate math functions.

The artificial path planning method is more efficient than the traditional ways. The result of our method is more useful in daily training. However, this is only the theory result. It is hard for ordinary athletics to understand. For any conditions, the result is shown in Figure.4a.

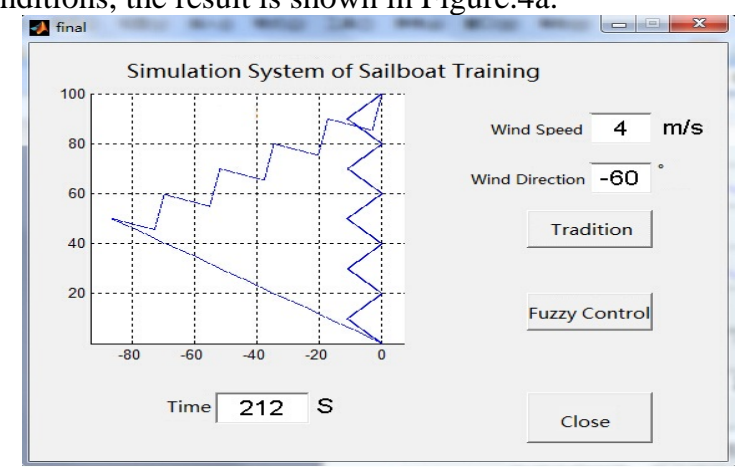

(a)

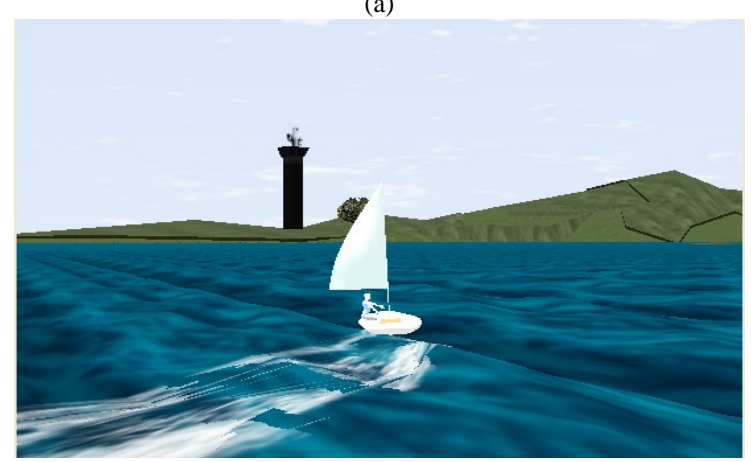

(b)

Figure 4. (a) The simulation of single sail training system (b) $3 \mathrm{D}$ visualization of single sailing

Based on our results above, we firstly use traditional method to calculate the experiment result as the knowledge storage. And then, use the divided thinking to optimize the shortest paths. In our paper, while using fuzzy control, we consider the sailing effective speed on the effective sailing directions and the distance away from the target. We take consider of these two factors to make the final decisions. 


\section{CONCLUSIONS}

In this paper we make a study on the simulation research of the single sailing, combining the traditional path planning algorithm with the intelligent path planning of the fuzzy control algorithm. According to the force analysis of sailing, the best sailing angle for the given environment is selected and the optimal path of the sailing during the sailing competition is planned out. Our research can provide the theoretical basis for scientific sailing training, as well as provide guidance and reference for improving the sailing competition results.

\section{ACKNOWLEDGEMENTS}

The research work is supported by the National Hi-tech Research and Program of China, 863 Program (No.2012AA090901). The corresponding author is Guojia Hou (hgjouc@gmail.com).

\section{REFERENCES}

[1] Ding Zurong, Hu Wenrong. The sailing and force analysis of the sailing [J]. Mechanics of medical biomechanics. 23 (3), pp.248-251, 2008.

[2] Wang fang. Study based on the analysis of ITTC-57 sailing power and motion simulation [D]. Qingdao: Qingdao university computer application technology, 2007.
[3] Sandler M, Wahl A, Zimmermann R, et al. Autonomous Guidance of Ships on Waterways [J]. Robotics and Autonomous System, 18(3),pp.327-335, 1996.

[4] GeYan. Sailing route planning based on fuzzy evolutionary theory research [D]. Qingdao: the ocean technology of The Ocean University of China, 2005.

[5] Xing Huili, Hu Xihou, GeYan. The optimization and simulation of sailing navigation path [J]. Computer engineering and application.

[6] Huang Junhui Fu Shenwen. The development and application of fuzzy control theory [J]. Information science and technology of China, 12,pp.303-306, 2006.

[7] Yao Zhang, Grant E.Hearn and Pratyush Sen. A Neural Network Approach to Ship Track-Keeping Control. IEEE Journal of ocean Engineering, 21(4), 1996.

[8] Wang Jinghua Zhang Cuimin. The path planning algorithm of intelligent robot applied in the Matlab implementation [J]. Journal of tian jin normal university in engineering, 16 (3),pp.47-50, 2006.

[9] GeYan, Meng Qingchun,Wei Zhengang, Dou Jinfeng, Zhang Wen. Research of sailing control method based on the Sugeno fuzzy control model [J]. Control and decision making, 19 (6)pp.659-662, 2004.

[10] Meng Qingchun,Zhuang Xiaodong YanBo, etc. The search method of robot path search in the dynamic environment based on fuzzy concept [J]. Robot, 23 (5),pp.397-355, 2001.

[11] GeYan, Meng Qingchun, et al. The optimal dynamic path planning method research of sailing along the straight line [J]. Control and decision making, 20 (12) ,pp.1360-1364, 2005.

[12] Xinghui Li. The optimal path planning method research of sailing around the buoy [D]. Qingdao: the computer application technology of The Ocean University of China, 2006. 